\section{(2) OPEN ACCESS}

\title{
Multiple breath washout (MBW) testing using sulfur hexafluoride: reference values and influence of anthropometric parameters
}

\author{
Frederik Trinkmann (1) , 1,2,3 Máté Maros @i] , ${ }^{3,4}$ Katharina Roth, ${ }^{1}$ Arne Hermanns, ${ }^{1}$ \\ Julia Schäfer, ${ }^{1}$ Joshua Gawlitza, ${ }^{5}$ Joachim Saur, ${ }^{1}$ Ibrahim Akin, ${ }^{1,6}$ Martin Borggrefe, ${ }^{1,6}$ \\ Felix J F Herth, ${ }^{2}$ Thomas Ganslandt ${ }^{3}$
}

\begin{abstract}
- Additional material for this paper are available online. To view these files, please visit the journal online (http://dx doi.org/10.1136/thoraxjnl2020-214717)
\end{abstract}

For numbered affiliations see end of article.

\section{Correspondence to} Dr Frederik Trinkmann, 1st Department of Medicine, University Medical Centre Mannheim, Mannheim, BadenWürttemberg, Germany; frederik.trinkmann@umm.de

An abstract containing preliminary analysis of the underlying data was presented at the online 2020 annual congress of the American Thoracic Society.

Received 27 February 2020 Revised 7 January 2021 Accepted 20 January 2021 Published Online First 16 February 2021

\begin{abstract}
Background Multiple breath washout (MBW) using sulfur hexafluoride $\left(\mathrm{SF}_{6}\right)$ has the potential to reveal ventilation heterogeneity which is frequent in patients with obstructive lung disease and associated small airway dysfunction. However, reference data are scarce for this technique and mostly restricted to younger cohorts. We therefore set out to evaluate the influence of anthropometric parameters on $\mathrm{SF}_{6}-\mathrm{MBW}$ reference values in pulmonary healthy adults.
\end{abstract}

Methods We evaluated cross-sectional data from 100 pulmonary healthy never-smokers and smokers (mean 51 (SD 20), range 20-88 years). Lung clearance index $(\mathrm{LCl})$, acinar $\left(\mathrm{S}_{\text {acin }}\right)$ and conductive $\left(\mathrm{S}_{\text {cond }}\right)$ ventilation heterogeneity were derived from triplicate $\mathrm{SF}_{6}-\mathrm{MBW}$ measurements. Global ventilation heterogeneity was calculated for the 2.5\% $\left(\mathrm{LCl}_{25}\right)$ and $5 \%\left(\mathrm{LCl}_{5}\right)$ stopping points. Upper limit of normal (ULN) was defined as the 95th percentile.

Results Age was the only meaningful parameter influencing SF - MBW parameters, explaining 47\% (Cl $33 \%$ to $59 \%$ ) of the variance in $\mathrm{LCl}, 32 \%(\mathrm{Cl} 18 \%$ to $47 \%)$ in $\mathrm{S}_{\text {acin }}$ and $10 \%(\mathrm{Cl} 2 \%$ to $22 \%)$ in $\mathrm{S}_{\text {cond }}$. Mean $\mathrm{LCl}$ increases from 6.3 (ULN 7.4) to 8.8 (ULN 9.9) in subjects between 20 and 90 years. Smoking accounted for $2 \%(\mathrm{Cl}$ $0 \%$ to $8 \%)$ of the variability in $\mathrm{LCl}, 4 \%(\mathrm{Cl} 0 \%$ to $13 \%)$ in $\mathrm{S}_{\text {acin }}$ and $3 \%(\mathrm{Cl} 0 \%$ to $13 \%)$ in $\mathrm{S}_{\text {cond }}$

Conclusion $\mathrm{SF}_{6}-\mathrm{MBW}$ outcome parameters showed an age-dependent increase from early adulthood to old age. The effect was most pronounced for global and acinar ventilation heterogeneity and smaller for conductive ventilation heterogeneity. No influence of height, weight and sex was seen. Reference values can now be provided for all important $\mathrm{SF}_{6}-\mathrm{MBW}$ outcome parameters over the whole age range.

Trial registration number NCT04099225.

\section{INTRODUCTION}

Multiple breath washout (MBW) has the potential to reveal ventilation heterogeneity which is frequently present in patients with obstructive lung disease and small airway dysfunction. However, it is often missed by commonly used tests. ${ }^{12}$ Spirometry was shown to be insensitive to changes in patients with asthma that can be made visible using $\mathrm{MBW}^{34}$ These changes are associated with symptoms, most notably dyspnoea, cough and phlegm in COPD. ${ }^{5}$

\section{Key messages}

What is the key question?

- Reference data for multiple breath washout (MBW) testing are scarce, with available studies being mostly restricted to younger cohorts and using nitrogen as tracer gas.

What is the bottom line?

- Sulfur hexafluoride-MBW-derived parameters show an age-dependent increase from early adulthood to old age, being most pronounced for global and acinar ventilation heterogeneity.

Why read on?

- For the first time, reference values can now be provided in an adult setting over a wide age range and for all common parameters.

Dyspnoea may be a direct expression of hyperinflation, ${ }^{6}$ which was shown to be addressable therapeutically. ${ }^{7}$ The impact of peripheral airways is yet not restricted to late stages. Instead small airway dysfunction occurs early in the natural course of the disease. In bronchial asthma, changes in small airways are also a hallmark and present in more than $90 \%$ of patients. Moreover, clinical subtypes of small airway disease were identified using different tests including $\mathrm{MBW} .{ }^{8}$ Lung clearance index (LCI) is the most frequently used MBW outcome parameter and refers to global ventilation heterogeneity. Phase III slopes represent local ventilation heterogeneity in the acinar $\left(\mathrm{S}_{\text {acin }}\right)$ and conductive $\left(\mathrm{S}_{\text {cond }}\right)$ airways. Open wash-in MBW using sulfur hexafluoride $\left(\mathrm{SF}_{6}\right)$ and mass spectrometry are considered the gold standard ${ }^{9}$ while being associated with high costs and effort. Hence, nitrogen $\left(\mathrm{N}_{2}\right)$ has been frequently used as tracer gas despite suffering from several methodological drawbacks such as back diffusion, ${ }^{10}{ }^{11}$ measurement inaccuracies ${ }^{12}$ and leaks. ${ }^{13}$ Introduction of a photo-magneto-acoustic multigas analyser allowed the direct measurement of $\mathrm{SF}_{6}$ concentrations with high accuracy. ${ }^{14}$ Recently, this led to construction of a closed-circuit $\mathrm{SF}_{6}-\mathrm{MBW}$ set-up considerably facilitating application and reducing costs. ${ }^{15}$ Meaningful differences in MBW outcome measures depending on the test gases have been described. ${ }^{16}{ }^{17}$ In general, $\mathrm{N}_{2}$-based
To cite: Trinkmann $\mathrm{F}_{\text {, }}$
Maros $\mathrm{M}$, Roth $\mathrm{K}$, et al.

Thorax 2021;76:380-386 
systems reproducibly yield higher absolute LCI readings than $\mathrm{SF}_{6}-\mathrm{MBW}^{18-20}$ To date, much clinical data are available in patients with cystic fibrosis using $\mathrm{N}_{2}$-based MBW systems. Reference data are scarce and mostly restricted to younger cohorts. Therefore, we set out to (1) evaluate the influence of anthropometric parameters on $\mathrm{SF}_{6}-\mathrm{MBW}$ outcome measures and (2) generate reference values in pulmonary healthy controls.

\section{METHODS}

\section{Subjects}

We evaluated cross-sectional data from pulmonary healthy neversmokers and smokers. Participants were included prospectively using convenience sampling. Subjects had normal lung function testing and no respiratory symptoms. Normal lung function required a combination of normal spirometry, whole-body plethysmography, impulse oscillometry and Krogh factor $\left(\mathrm{K}_{\mathrm{CO}}\right)$ in both qualitative and quantitative analyses. Details are given in the online supplemental appendix 1 . The study was registered at ClinicalTrials.gov. Written informed consent was obtained from all participants prior to inclusion.

\section{Study protocol}

All subjects underwent three consecutive MBW tests in upright position followed by impulse oscillometry (MasterScreen IOS, CareFusion 234, Höchberg, Germany), spirometry and wholebody plethysmography (MasterScreen Body). Oscillometry was performed during tidal breathing. Parameters were averaged over multiple respiratory cycles within $20 \mathrm{~s}$. Transfer factor for carbon monoxide (TLCO) was derived from $\mathrm{K}_{\mathrm{CO}}$ and ventilated alveolar volume as measured in single breath technique. All lung function measurements were taken cross-sectionally at a single time point and independently assessed by two experienced investigators. Reference values for spirometry, whole-body plethysmography and transfer factors were derived from the revised 1993 version of the European Community for Steel and Coal (ECSC) equations. ${ }^{21}$ For impulse oscillometry, reference values as provided by Vogel and Smidt ${ }^{22}$ were used.

\section{Multiple breath washout}

A commercially available closed-circuit system (Innocor, PulmoTrace ApS, Glamsbjerg, Denmark) was used for MBW measurements. ${ }^{15}$ The device consists of a $3 \mathrm{~L}$ rebreathing bag filled with a mixture of room air and test gas $\left(94 \% \mathrm{O}_{2}, 1 \% \mathrm{SF}_{6}\right.$ and $5 \%$ $\mathrm{N}_{2} \mathrm{O}$; PulmoTrace ApS) from a commercially available on-board gas cylinder (bolus fraction 20\%). Subjects breathed through the device using a mouthpiece and wearing a nose clip. A scrubber was serially placed between the rebreathing bag and the patient to reduce carbon dioxide concentrations during rebreathing. A standard bag volume of $2.5 \mathrm{~L}$ was used. Adjustments were made upfront in steps of 0.25 to $0.5 \mathrm{~L}$, if functional residual capacity (FRC) was known or suspected to considerably deviate. Likewise, adaptions became necessary during measurements with the subject emptying the bag early during rebreathing. Maximal slow inspirations up to the bag volume followed by slow expirations were performed for the first six breaths in order to achieve a complete wash-in. Inspiratory gas concentrations were closely monitored and held below 3\% for carbon dioxide and above $18 \%$ for oxygen. The further manoeuvre was performed during relaxed tidal breathing. Washout was then started by switching between the bag and room air using a fast-operating pneumatic valve. This was automatically triggered at the end of expiration under the direction of the operator. We stopped the test when end tidal $\mathrm{SF}_{6}$ had fallen below $1 / 40$ of the starting concentration for three consecutive breaths. Detailed analysis and quality control were performed offline using proprietary software provided by the manufacturer (V.8.0 beta 1 software). Mean FRC and LCI were derived from three consecutive washouts during tidal breathing. LCI is defined as the number of FRC turnovers to reduce tracer gas to a predefined level. This is referred to as stopping point and was calculated for the traditional $2.5 \%\left(\mathrm{LCI}_{2.5}\right)$ as well as $5 \%\left(\mathrm{LCI}_{5}\right)$ of the initial tracer gas concentration. Additionally, phase III slopes were analysed from multiple breaths yielding parameters of acinar $\left(S_{\text {acin }}\right)$ and conductive $\left(\mathrm{S}_{\text {cond }}\right)$ ventilation heterogeneity. We used a semiautomated approach plotting slopes against FRC turnovers. All breaths were visually analysed and excluded if no clear linear phase III portion could be identified. $S_{\text {cond }}$ was then derived by linear regression in the range between 1.5 and 6 turnovers. $S_{\text {acin }}$ was calculated as the mean slope of the three first breaths minus $\mathrm{S}_{\text {cond }}$ contribution. ${ }^{9}$ Only subjects with at least two technically acceptable MBW measurements ${ }^{23}$ based on slightly modified American Thoracic Society (ATS)/ European Respiratory Society (ERS) criteria (online supplemental appendix 1) were included in the final analysis.

\section{Statistical analysis}

Mean values are given as \pm SD unless stated otherwise. Student's t-test or $\chi^{2}$ test was used to compare continuous or categorical variables, respectively. Cohen's d with $95 \% \mathrm{CI}$ is given as absolute value to quantify the effect size (ES). The coefficient of variation $(\mathrm{CV})$ was calculated as $\mathrm{SD} /$ mean from the valid MBW measurements. An alpha error of less than 5\% in twosided testing was considered statistically significant. R statistical software (V.3.5.1, Foundation for Statistical Computing, Vienna, Austria) was used for all data analyses. ${ }^{24}$ First, the influence of anthropometric parameters was assessed. Age, height, weight and sex were included into an elastic-net generalised linear model assessed at lambda plus $1 \mathrm{SE}$. L1 regularisation was used

Table 1 Baseline characteristics

\begin{tabular}{|c|c|c|c|c|c|c|c|c|c|}
\hline & \multirow[b]{2}{*}{ Unit } & \multicolumn{2}{|l|}{$\begin{array}{l}\text { Overall } \\
(\mathrm{N}=100)\end{array}$} & \multicolumn{2}{|c|}{$\begin{array}{l}\text { Never-smoker } \\
(n=68)\end{array}$} & \multicolumn{2}{|l|}{$\begin{array}{l}\text { Smoker } \\
(\mathrm{n}=32)\end{array}$} & \multirow[b]{2}{*}{$P$ value } & \multirow[b]{2}{*}{ ES } \\
\hline & & Value & Range & Value & Range & Value & Range & & \\
\hline Age & Years & $51 \pm 20$ & $20-88$ & $49 \pm 21$ & $20-88$ & $54 \pm 16$ & $22-81$ & 0.22 & 0.24 (0.19 to 0.66$)$ \\
\hline Height & $\mathrm{cm}$ & $171 \pm 9$ & 152-198 & $172 \pm 9$ & 153-198 & $169 \pm 9$ & 152-193 & 0.22 & 0.26 (-0.16 to 0.69$)$ \\
\hline Weight & $\mathrm{kg}$ & $76 \pm 17$ & $45-132$ & $75 \pm 16$ & $45-132$ & $79 \pm 20$ & $51-120$ & 0.33 & $0.23(-0.19$ to 0.65$)$ \\
\hline BMI & $\mathrm{kg} / \mathrm{m}^{2}$ & $26.2 \pm 5.4$ & $16.3-49.1$ & $25.6 \pm 5.2$ & $16.3-49.1$ & $27.5 \pm 5.5$ & $19.9-37.8$ & 0.11 & 0.35 (-0.08 to 0.78$)$ \\
\hline Male & $\mathrm{n}(\%)$ & $54(54)$ & & $37(54)$ & & $17(53)$ & & 1.0 & $0.03(-0.39$ to 0.45$)$ \\
\hline
\end{tabular}

Effect size (ES) as measured by Cohen's $d$ with $95 \% \mathrm{Cl}$.

BMI, body mass index. 
for variable selection based on a k-fold cross-validation approach to find optimal hyperparameters. In order to quantify the influence of these anthropometric variables and smoking status on MBW parameters, a standard generalised linear model with Gaussian link function without regularisation was used. Their corresponding CIs of the explained variances were estimated based on 10000 bootstrap samples with replacement for sample sizes of 100. Second, reference values were calculated based on a cubic splines generalised additive model for location scale and shape (GAMLSS) approach. Separate models were fitted (1) independently of smoking status and (2) for never-smokers only. ${ }^{25}$ Upper limit of normal (ULN) and lower limit of normal (LLN) were defined as the 95 th and 5 th percentiles, respectively.

\section{RESULTS}

\section{Baseline characteristics and lung function}

Data were acquired in a total of 104 subjects. The prespecified exclusion criteria were met by four subjects, resulting in 100 subjects (54 men) undergoing final analysis. The baseline characteristics of 68 never-smokers and 32 smokers $(5$ active smokers, 27 former smokers) are summarised with betweengroup differences in table 1 . Lung function data are given in table 2. Differences between smokers and never-smokers were only found for maximum expiratory flow at 25\% $\left(\mathrm{MEF}_{25}\right)$ of FVC and $\mathrm{S}_{\text {acin }}$. The overall CV was 3.2\% for $\mathrm{LCI}_{2.5}$ and $3.3 \%$ for $\mathrm{LCI}_{5}$. At least two valid phase III slope measurements could not be acquired in 10 subjects. These were slightly older $(55 \pm 20$ vs $50 \pm 20$ years, $\mathrm{p}=0.46, \mathrm{ES}=0.26, \mathrm{CI}-0.40$ to 0.91 ) as compared with subjects with successful measurements. Additionally, phase III slopes showed larger variability with a CV of $50 \%$ for $\mathrm{S}_{\text {acin }}$ and $124 \%$ for $S_{\text {cond }}$. The mean washout duration was $104 \pm 37 \mathrm{~s}$ for $\mathrm{LCI}_{2.5}$ and $79 \pm 27 \mathrm{~s}$ for $\mathrm{LCI}_{5}(\mathrm{p}<0.0001, \mathrm{ES}=0.8, \mathrm{CI} 0.5$ to 1.1$)$.

\section{Influence of anthropometric parameters}

Age was the only meaningful contributor to LCI and $\mathrm{S}_{\text {acin }}$ in the regularised model, whereas no association with $S_{\text {cond }}$ was found in the overall collective. In a non-penalised model, age explained $47 \%$ (CI $33 \%$ to $59 \%$ ) of the variance in LCI, 32\% (CI 18\% to $47 \%$ ) in $\mathrm{S}_{\text {acin }}$ and $10 \%$ (CI $2 \%$ to $22 \%$ ) in $\mathrm{S}_{\text {cond }}$. Smoking accounted for $2 \%$ (CI $0 \%$ to $8 \%$ ) of the variability in LCI, $4 \%$

\begin{tabular}{|c|c|c|c|c|c|c|c|c|c|}
\hline & \multirow[b]{2}{*}{ Unit } & \multicolumn{2}{|l|}{$\begin{array}{l}\text { Overall } \\
(\mathrm{N}=100)\end{array}$} & \multicolumn{2}{|c|}{$\begin{array}{l}\text { Never-smoker } \\
(\mathrm{n}=68)\end{array}$} & \multicolumn{2}{|l|}{$\begin{array}{l}\text { Smoker } \\
(n=32)\end{array}$} & \multirow[b]{2}{*}{$P$ value } & \multirow[b]{2}{*}{ ES } \\
\hline & & Value & Range & Value & Range & Value & Range & & \\
\hline \multicolumn{10}{|l|}{ Spirometry } \\
\hline $\mathrm{FEV}_{1} / \mathrm{FVC}$ & $\%$ & $83 \pm 6$ & 70-107 & $83 \pm 6$ & 70-107 & $82 \pm 5$ & $71-93$ & 0.58 & $0.11(-0.31$ to 0.53$)$ \\
\hline $\mathrm{FEV}_{1}$ & $\%$ pred & $103 \pm 14$ & $65-135$ & $103 \pm 14$ & $73-135$ & $103 \pm 13$ & $65-124$ & 0.87 & $0.04(-0.38$ to 0.46$)$ \\
\hline VC & $\%$ pred & $104 \pm 14$ & $64-138$ & $103 \pm 15$ & $64-138$ & $105 \pm 13$ & $73-131$ & 0.54 & $0.13(-0.30$ to 0.55$)$ \\
\hline $\mathrm{MEF}_{75}$ & \%pred & $98 \pm 23$ & $48-164$ & $98 \pm 24$ & 49-164 & $97 \pm 21$ & $48-138$ & 0.83 & $0.04(-0.38$ to 0.46$)$ \\
\hline $\mathrm{MEF}_{50}$ & \%pred & $90 \pm 29$ & $35-170$ & $92 \pm 30$ & $37-170$ & $86 \pm 26$ & $35-151$ & 0.33 & $0.20(-0.22$ to 0.62$)$ \\
\hline $\mathrm{MEF}_{25}$ & $\%$ pred & $65 \pm 26$ & $13-141$ & $68 \pm 26$ & $18-141$ & $58 \pm 25$ & $13-111$ & $<0.05^{*}$ & $0.43(-0.01$ to 0.86$)$ \\
\hline \multicolumn{10}{|c|}{ Body plethysmography } \\
\hline TLC & $\%$ pred & $110 \pm 15$ & $81-157$ & $109 \pm 14$ & $81-140$ & $111 \pm 16$ & $84-157$ & 0.48 & $0.16(-0.27$ to 0.58$)$ \\
\hline RV & $\%$ pred & $126 \pm 36$ & $13-219$ & $125 \pm 36$ & $15-219$ & $127 \pm 39$ & $13-213$ & 0.80 & $0.06(-0.36$ to 0.48$)$ \\
\hline $\mathrm{RV} / \mathrm{TLC}$ & $\%$ & $39 \pm 9$ & $20-57$ & $38 \pm 9$ & $20-57$ & $40 \pm 8$ & $20-54$ & 0.28 & $0.22(-0.20$ to 0.65$)$ \\
\hline$s R_{\text {tot }}$ & \%pred & $73 \pm 32$ & $22-178$ & $72 \pm 31$ & $22-178$ & $75 \pm 33$ & $26-154$ & 0.65 & $0.10(-0.32$ to 0.52$)$ \\
\hline \multicolumn{10}{|l|}{ Gas transfer } \\
\hline $\mathrm{K}_{\mathrm{co}}$ & \%pred & $96 \pm 11$ & $80-135$ & $97 \pm 12$ & $80-135$ & $93 \pm 9$ & $80-114$ & 0.12 & $0.31(-0.12$ to 0.73$)$ \\
\hline TLCO & \%pred & $87 \pm 12$ & $58-134$ & $87 \pm 13$ & $65-134$ & $85 \pm 10$ & $58-106$ & 0.34 & $0.19(-0.24$ to 0.61$)$ \\
\hline \multicolumn{10}{|c|}{ Impulse oscillometry } \\
\hline R5 & $\%$ pred & $96 \pm 20$ & $53-144$ & $95 \pm 20$ & 53-137 & $98 \pm 19$ & $57-144$ & 0.43 & $0.17(-0.26$ to 0.59$)$ \\
\hline D5-20 & $\%$ & $15 \pm 14$ & $0-57$ & $14 \pm 14$ & $0-57$ & $17 \pm 12$ & $0-52$ & 0.21 & $0.26(-0.17$ to 0.68$)$ \\
\hline Ax & $\mathrm{kPa} / \mathrm{L}$ & $0.32 \pm 0.30$ & $0.0-1.42$ & $0.31 \pm 0.33$ & $0.0-1.42$ & $0.32 \pm 0.24$ & $0.03-1.35$ & 0.96 & $0.01(-0.42$ to 0.44$)$ \\
\hline $\mathrm{F}_{\text {res }}$ & $\mathrm{Hz}$ & $12 \pm 4$ & $3-23$ & $11 \pm 4$ & $3-23$ & $12 \pm 3$ & $7-19$ & 0.27 & $0.22(-0.21$ to 0.64$)$ \\
\hline \multicolumn{10}{|c|}{ Multiple breath washout } \\
\hline $\mathrm{LCl}_{2.5}$ & - & $7.2 \pm 1.0$ & $5.7-10.2$ & $7.1 \pm 0.9$ & $5.7-10.2$ & $7.4 \pm 1.0$ & $5.9-9.9$ & 0.07 & $0.41(-0.02$ to 0.84$)$ \\
\hline $\mathrm{LCl}_{5}$ & - & $5.6 \pm 0.7$ & $4.5-7.4$ & $5.6 \pm 0.7$ & $4.5-7.4$ & $5.8 \pm 0.6$ & $4.7-7.2$ & 0.08 & $0.39(-0.05$ to 0.81$)$ \\
\hline $\mathrm{FRC}_{\mathrm{MBW}}$ & L & $2.9 \pm 0.7$ & $1.6-4.6$ & $3.0 \pm 0.7$ & $1.6-4.6$ & $2.8 \pm 0.7$ & $1.9-4.3$ & 0.37 & $0.19(-0.61$ to 0.24$)$ \\
\hline $\mathrm{S}_{\text {acin }}$ & $\mathrm{L}^{-1}$ & $0.09 \pm 0.07$ & -0.04 to 0.36 & $0.08 \pm 0.06$ & -0.04 to 0.28 & $0.11 \pm 0.08$ & -0.04 to 0.36 & $<0.05^{*}$ & 0.54 (0.07 to 1.00$)$ \\
\hline $\mathrm{S}_{\text {cond }}$ & $\mathrm{L}^{-1}$ & $0.04 \pm 0.04$ & -0.07 to 0.2 & $0.04 \pm 0.04$ & -0.05 to 0.2 & $0.04 \pm 0.05$ & -0.07 to 0.16 & 0.78 & $0.07(-0.38$ to 0.51$)$ \\
\hline
\end{tabular}

Effect size (ES) as measured by Cohen's d with $95 \% \mathrm{Cl}$.

*Statistically significant at $p<0.05$ (never-smoker vs smoker).

$\mathrm{Ax}$, area under reactance curve; D5-20, frequency dependence of resistance; FRC, functional residual capacity; $\mathrm{F}_{\text {res }}$, resonance frequency; $\mathrm{K}_{\text {co, }}$ Krogh factor; $\mathrm{LCl}$, lung clearance index at 2.5\% and 5\% stopping points; MBW, multiple breath washout; MEF, maximum expiratory flow at 75\%, 50\% and 25\% of FVC; $\%$ pred, percent of predicted; R5,

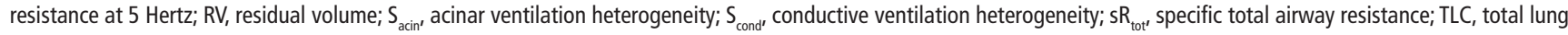
capacity; TLCO, transfer factor for carbon monoxide; VC, vital capacity. 
A

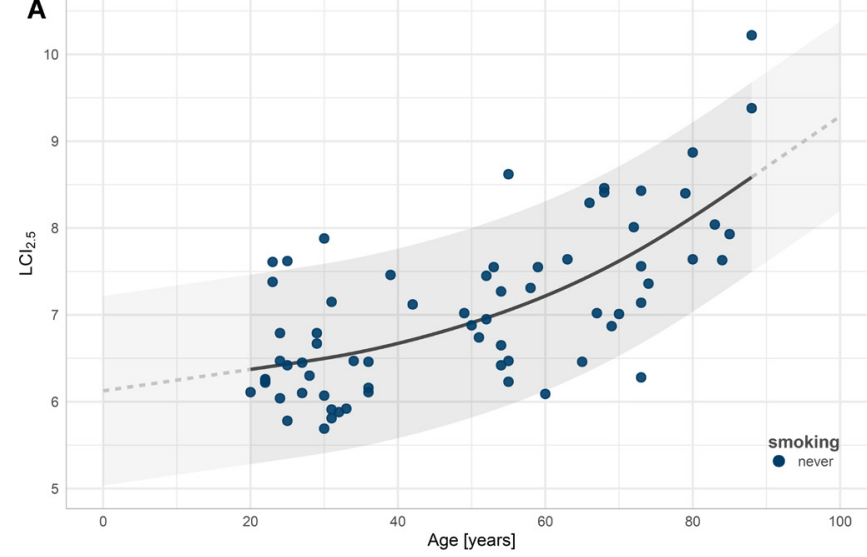

B

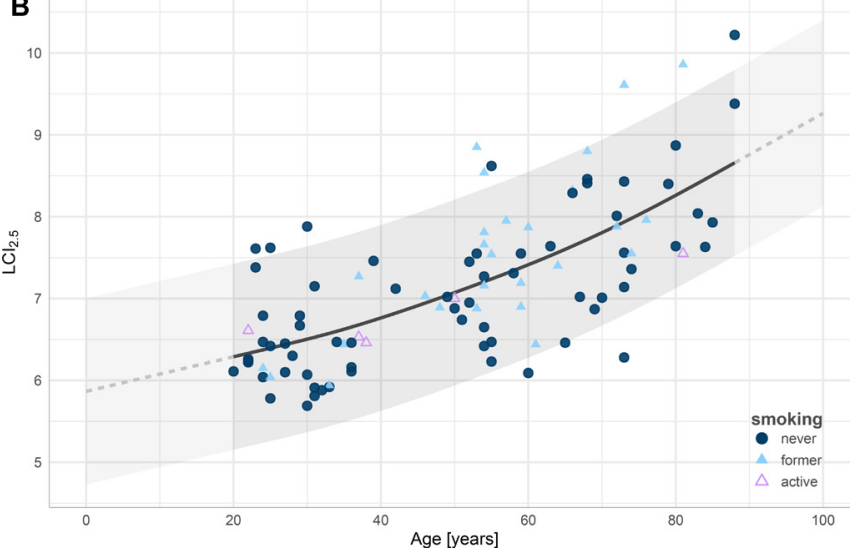

Figure $1 \quad \mathrm{LCl}_{2.5}$ normal values. Age-dependent plot of predicted values (solid line) with ULN and LLN. (A) Never-smokers $(n=68)$ provide overall lower predictions as compared with (B) additionally including former or active smokers $(n=100)$. Dashed lines and lighter grey represent a model-based extrapolation of actually measured cross-sectional data. $\mathrm{LCl}_{2.5^{\prime}}$ lung clearance index at $2.5 \%$ stopping point; $\mathrm{LLN}$, lower limit of normal; ULN, upper limit of normal.

(CI $0 \%$ to $13 \%$ ) in $\mathrm{S}_{\text {acin }}$ and $3 \%$ (CI $0 \%$ to $13 \%$ ) in $\mathrm{S}_{\text {cond }}$, respectively. We therefore calculated normal values with ULN and LLN corrected for age.

\section{Reference values}

Figure 1 gives age-dependent predictions for $\mathrm{LCI}_{25}$ in the overall collective and never-smokers only. Likewise, predicted values for phase III slopes are given in figure 2. The underlying models are available for download from GitHub (https:/github.com/ ftrinkmann). A summary of calculated reference values per decade is given in table 3. Age-dependent predictions for $\mathrm{LCI}_{5}$ in the overall collective and never-smokers can be found in online supplemental e-Figure 1.

\section{DISCUSSION}

We found that age is the only anthropometric parameter contributing to $\mathrm{SF}_{6}-\mathrm{MBW}$ outcome measures. The effect was most pronounced for global and acinar ventilation heterogeneity and smaller for conductive ventilation heterogeneity. No influence of height, weight and sex was seen in our adult population. On this basis, we were able to generate age-corrected reference values for $\mathrm{SF}_{6}-\mathrm{MBW}$ ranging from early adulthood to old age.

Our results extend the data available for paediatric cohorts and are in accordance with previous findings. Lum and coworkers $^{26}$ could demonstrate that LCI decreases with height during the first approximately 6 years of life and then remains rather stable after reaching around $115 \mathrm{~cm}$. Hence, they proposed to use a constant ULN of 7.56 defined by the 97.5 th percentile in subjects older than 6 years, which corresponds well to our model. No correlation between age and LCI was found when including younger collectives. ${ }^{27} 28$ With the normal range appearing to remain stable for the first four decades of life, a fixed ULN of 6.8 and 7.0 was proposed by Horsley et $a l^{28}$ and Fuchs et al. ${ }^{29}$ In patients older than 40 years a larger dispersion was seen. Despite all of these approaches being based on $\mathrm{SF}_{6}$, small differences may be partly explained by system set-ups and tracer gas concentrations. Considerably larger differences can be attributed to tracer gases themselves as compared with setup. It is well known that $\mathrm{N}_{2}$-MBW reproducibly yields higher absolute LCI readings than $\mathrm{SF}_{6}-\mathrm{MBW} .{ }^{17}{ }^{18}$ Much of this difference can be explained by physiological properties and technical aspects associated with the test gases. ${ }^{9} 121719$ Concerning local ventilation heterogeneity, age was found to explain $7 \%$ and $16 \%$ of the variability in $\mathrm{S}_{\text {acin }}$ and $\mathrm{S}_{\text {cond }}$ derived from $\mathrm{N}_{2}-\mathrm{MBW}$, respectively. ${ }^{30}$ Moreover, a significant influence of sex on $S_{\text {acin }}$ as well as age on LCI was found. This corresponds to our data set where $10 \%$ of the variability in $\mathrm{S}_{\text {cond }}$ is explained by age. In contrast, contribution for LCI and $\mathrm{S}_{\text {acin }}$ is higher in our collective with $47 \%$ and $32 \%$. Reference intervals for parameters of local ventilation heterogeneity using $\mathrm{N}_{2}$ as tracer gas were higher than those found for $\mathrm{SF}_{6}$ in our data at hand. Interestingly, mean LCI reference values were lower in their investigation than those for $\mathrm{SF}_{6}-\mathrm{MBW}$. This is contradictory to our findings and previously published data. While $\mathrm{N}_{2}$ readings were found to be consistently higher in general as discussed above, Houltz and coworkers ${ }^{31}$ could demonstrate higher mean predictions for LCI over a wide range between 7 and 70 years. Verbanck and coworkers found a 0.22-unit change in LCI per decade, while we could demonstrate an increasing rate with age between 0.2 and 0.5 units per decade. ${ }^{30}$ It should be noted that including smokers led to a 0.3 increase in mean LCI, corresponding to a 10 -year effect of ageing. In addition to differences in test gases and set-ups, parts of these differences may be explained by the sequence of lung function tests. Potential airway dilatation may lead to distortion of tidal breathing techniques. Therefore, we conducted all examinations requiring tidal breathing strictly before forced manoeuvres. Taken together, either test gas requires separate reference data and individual values should not be used interchangeably.

When interpreting our results, several aspects should be taken into consideration. For $\mathrm{S}_{\text {acin }}$ and $\mathrm{MEF}_{25}$, significant differences were found between smokers and only never-smokers, respectively. For generating reference values, it would have been desirable to strictly include only never-smokers. However, this would have narrowed the age range investigated considerably as finding otherwise healthy, never-smoking septuagenarians and octogenarians is particularly difficult. We therefore applied an extremely strict definition for controls. This did not only include commonly used history, respiratory symptoms and spirometric findings. Additionally, we required parameters from wholebody plethysmography, oscillometry and gas transfer to be within the normal range for both never-smoking and smoking controls, respectively. As a consequence, smoking status explains a maximum of $4 \%$ of the variability in MBW parameters. Therefore, calculation of normal values itself is hardly affected by smoking status using our GAMLSS approach.

Further, it should be noted that a solid definition of normality is difficult especially in elderly patients. This is important in the context of the rather broad range of residual volume/total lung capacity (RV/TLC) (20\%-57\%) and TLCO (58\%-134\% 

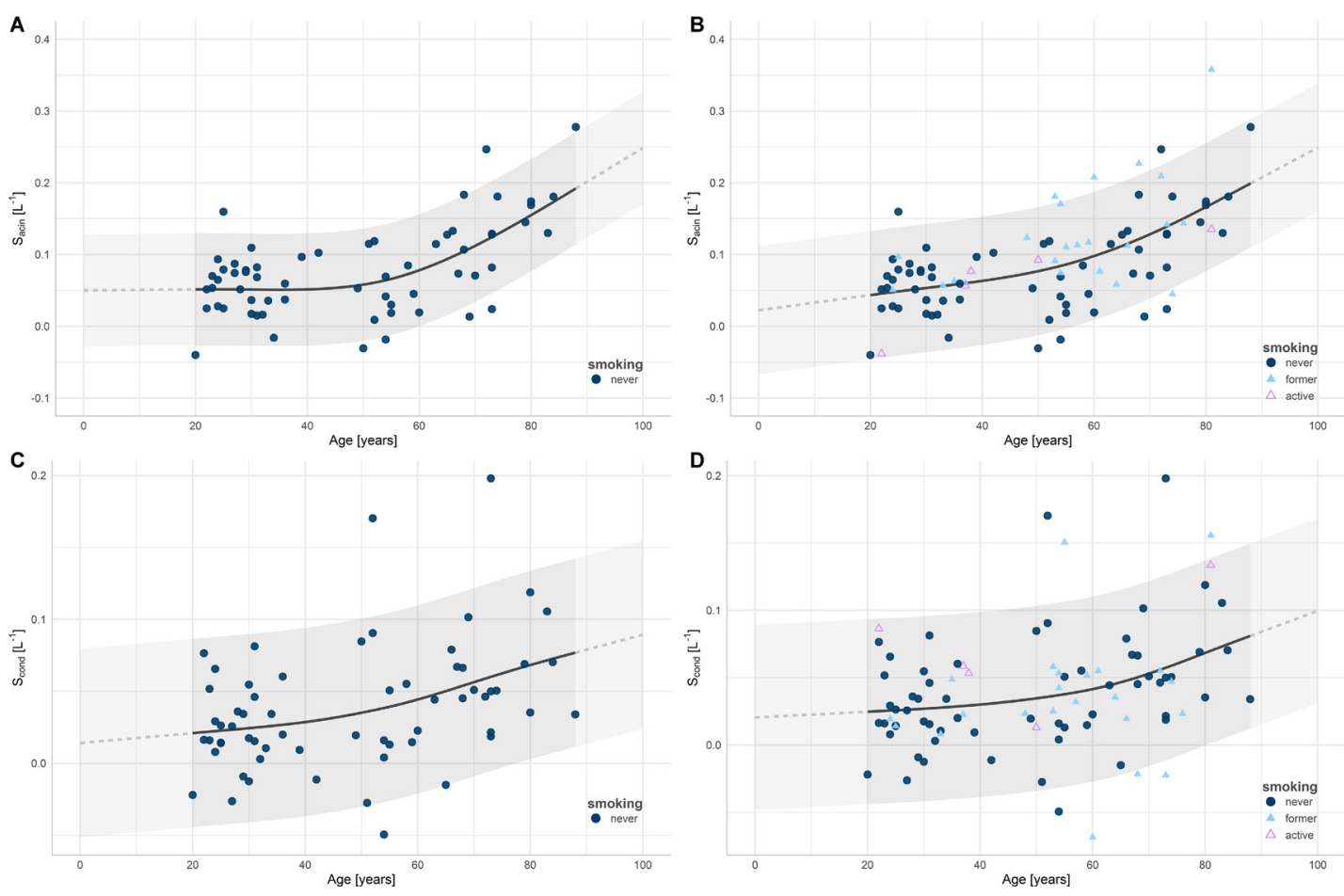

Figure 2 Phase III slope normal values. Age-dependent plot of predicted values (solid line) with ULN and LLN. (A) Never-smokers ( $n=68$ ) provide overall lower predictions and less age dependency as compared with (B) additionally including former or active smokers for $S_{\text {acin }}(n=100)$. (C) Marginal differences were found for $S_{\text {cond }}$ in never-smokers $(n=68)$ as compared with $(D)$ additionally including former or active smokers $(n=100)$. Dashed lines and lighter grey represent a model-based extrapolation of actually measured cross-sectional data. LLN, lower limit of normal; $\mathrm{S}_{\text {acin' }}$ acinar ventilation heterogeneity; $S_{\text {cond' }}$ conductive ventilation heterogeneity; ULN, upper limit of normal.

of predicted) found in our study. An increased RV/TLC as well as differences between TLCO and $\mathrm{K}_{\mathrm{CO}}$ are suggestive of ventilation heterogeneity and air-trapping. However, most of the values at the upper end (RV/TLC) or lower end (TLCO) were found in subjects well above 70 years of age. While reference data for body plethysmography are scarce in this age group, none of the subjects reported respiratory symptoms nor showed abnormal values in standard diagnostics. Moreover, we did not find disproportional deviations for ventilation heterogeneity, peripheral resistance or elastic properties. Taken together, this lets us assume that ageing effects within the physiological range

Table 3 Calculated reference values per decade

\begin{tabular}{|c|c|c|c|c|c|c|c|c|}
\hline \multirow[b]{2}{*}{ Age (years) } & \multicolumn{2}{|c|}{$\mathrm{LCl}_{2.5}(-)$} & \multicolumn{2}{|c|}{$\mathrm{LCl}_{5}(-)$} & \multicolumn{2}{|c|}{$S_{\text {acin }}\left(L^{-1}\right)$} & \multicolumn{2}{|c|}{$\mathrm{S}_{\text {cond }}\left(\mathrm{L}^{-1}\right)$} \\
\hline & Mean & ULN & Mean & ULN & Mean & ULN & Mean & ULN \\
\hline 10 & 6.1 & 7.2 & 4.9 & 5.7 & 0.03 & 0.12 & 0.02 & 0.09 \\
\hline 20 & 6.3 & 7.4 & 5.0 & 5.8 & 0.04 & 0.13 & 0.02 & 0.09 \\
\hline 30 & 6.5 & 7.6 & 5.2 & 6.0 & 0.05 & 0.14 & 0.03 & 0.10 \\
\hline 40 & 6.8 & 7.9 & 5.3 & 6.1 & 0.06 & 0.15 & 0.03 & 0.10 \\
\hline 50 & 7.1 & 8.2 & 5.6 & 6.4 & 0.08 & 0.17 & 0.03 & 0.10 \\
\hline 60 & 7.4 & 8.5 & 5.8 & 6.6 & 0.10 & 0.19 & 0.04 & 0.11 \\
\hline 70 & 7.8 & 8.9 & 6.1 & 6.9 & 0.13 & 0.22 & 0.05 & 0.12 \\
\hline 80 & 8.3 & 9.4 & 6.3 & 7.1 & 0.17 & 0.26 & 0.07 & 0.14 \\
\hline 90 & 8.8 & 9.9 & 6.6 & 7.4 & 0.21 & 0.30 & 0.08 & 0.15 \\
\hline 100 & 9.3 & 10.4 & 6.9 & 7.7 & 0.25 & 0.33 & 0.10 & 0.17 \\
\hline
\end{tabular}

$\mathrm{LCl}$, lung clearance index at $2.5 \%$ and $5 \%$ stopping points; $\mathrm{S}_{\text {acin }}$ acinar ventilation heterogeneity; $\mathrm{S}_{\text {cond' }^{\prime}}$ conductive ventilation heterogeneity; ULN, upper limit of normal. rather than unrecognised disease can be considered responsible for these changes.

Although age was not associated with $\mathrm{S}_{\text {cond }}$ in the regularised model, we decided to also provide age-corrected reference values since it explains as much as $10 \%$ of the variance. $S_{\text {cond }}$ is primarily subject to heterogeneity in pressure volume characteristics and bronchomotor tone of conductive airways. ${ }^{32}$ In contrast, $S_{\text {acin }}$ is heavily depending on branching asymmetry as well as parallel variability of the acini. ${ }^{33}$ This does not only explain differences between subjects, diseases or risk factors. Also, the different behaviour of ageing on $S_{\text {acin }}$ and $S_{\text {cond }}$ found in our investigation may be attributable to these factors. This is substantiated by previous findings. Considerable effects of ageing were described on airflow dynamics in a computational model. ${ }^{34}$ These correspond to ventilation defects found on imaging in otherwise healthy elderly subjects. ${ }^{35}$ However, no longitudinal data are currently available for functional tests and should therefore be subject of further investigation.

In contrast to the high intratest and intertest variability of traditional parameters of small airway obstruction such as MEF, ${ }^{3637}$ LCI was shown to be highly reproducibly. ${ }^{23}$ Variability of phase III slopes is larger as compared with the CV of up to $34 \%$ shown for MEF. ${ }^{37}$ Clinical implications of these have not been conclusively investigated to date. Nevertheless, impairment of $S_{\text {acin }}$ in patients with pulmonary hypertension allows to assume that early changes can already be detected and tracked today. ${ }^{38}$ In smokers with normal spirometry, changes in $S_{\text {cond }}$ were found and improvements to normal remained 12 months after smoking cessation. ${ }^{39}$ These findings are supported by data in patients with cystic fibrosis ${ }^{40}$ and adults with asthma. ${ }^{41} \mathrm{~S}_{\text {cond }}$ and $\mathrm{S}_{\text {acin }}$ may not represent all ventilation heterogeneities covered by LCI. Vice versa, LCI may not represent all ventilation heterogeneities 
covered by either $S_{\text {cond }}$ or $S_{\text {acin }}{ }^{42}$ Therefore, a combined interpretation of parameters representing global and local ventilation heterogeneity seems promising. In contrast, MEF-derived parameters were shown to not contribute usefully to clinical decision making. ${ }^{43}$

Total test duration is important in MBW testing. In general, washout is time-critical in $\mathrm{N}_{2}-\mathrm{MBW}$, whereas wash-in and costs are relevant factors in $\mathrm{SF}_{6}-\mathrm{MBW}$. Shorter washout durations are therefore an advantage of $\mathrm{SF}_{6}$ allowing successful measurements also in patients with severe obstructive lung disease. ${ }^{41}$ Nevertheless, it should be noted that requiring a wash-in phase may neutralise some of the advantages of $\mathrm{SF}_{6}$. For both tracer gases, several adaptions to the measurement protocols have been proposed, including reduction of total measurements or earlier cut-offs for terminating the washout. ${ }^{4-47}$ Moreover, closed-circuit SF -MBW was shown to effectively reduce wash-in times. ${ }^{15}$ We therefore calculated reference values for an earlier 5\% stopping point reducing washout times considerably by $24 \%$. Previously, an additional $34 \%$ reduction of total test time was achieved when only conducting two technically acceptable measurements. ${ }^{23}$ Together these adaptions may increase success rates in general. However, it should be noted that a comparative evaluation of clinical endpoints and diagnostic performance is warranted especially concerning abbreviated washout.

Our data considerably extend the current knowledge in adult $\mathrm{SF}_{6}-\mathrm{MBW}$ testing. Reference values can now be provided over the whole age range. For the first time in adult MBW testing, this is not only restricted to the most common parameter LCI, but also includes phase III slopes $S_{\text {acin }}$ and $S_{\text {cond }}$. While extrapolations of our model fit well to available data for subjects older than 6 years, normative data for phase III parameters that can be used for comparison are overall scarce. We were able to acquire local ventilation heterogeneity parameters in a considerable number of subjects but also encountered some difficulties. Analysis was not successful in 10 subjects. This was most often due to either not having sufficient phase III to compute slopes or an intermittently irregular breathing pattern. Together with the overall variability of these analyses, further research is warranted especially concerning clinical outcome studies. Our findings were acquired exclusively in white European adults sharing the lack of different ethnicities as a major drawback of most cohorts. Nevertheless, our model provides a sound basis for calculation of normal values for both research settings and clinical routine. Finally, two methodological considerations should also be taken into account. First, reference values for conventional lung function tests were derived from ECSC equations. ${ }^{21}$ Second, we used commercially available gas cylinders. These also contain $\mathrm{N}_{2} \mathrm{O}$, which is identical to the mixture used for cardiac output determination while differing in bolus fraction. ${ }^{148}$ Although $\mathrm{N}_{2} \mathrm{O}$ is dispensable for $\mathrm{MBW}$ measurements, we were not able to use an $\mathrm{N}_{2} \mathrm{O}$-free mixture due to lack of availability.

\section{CONCLUSIONS}

$\mathrm{SF}_{6}$-MBW parameters showed an age-dependent increase from early adulthood to old age. The effect was most pronounced for global and acinar ventilation heterogeneity. A smaller yet still considerable influence was seen for conductive ventilation heterogeneity. No influence of other anthropometric parameters such as height, weight and sex was found. Together with previous findings, reference values for LCI can now be provided over the whole age range as well as for an earlier 5\% stopping point. Moreover, our data are not restricted to global ventilation heterogeneity but also comprise the parameters of local ventilation heterogeneities $\mathrm{S}_{\text {acin }}$ and $\mathrm{S}_{\text {cond }}$.

\section{Author affiliations}

${ }^{1} 1$ st Department of Medicine, University Medical Centre Mannheim, Mannheim, Baden-Württemberg, Germany

${ }^{2}$ Department of Pneumology and Critical Care Medicine, Thoraxklinik at University Hospital Heidelberg, Translational Lung Research Centre Heidelberg (TLRC), Member of German Centre for Lung Research (DZL), Heidelberg, Baden-Württemberg, Germany

${ }^{3}$ Department of Biomedical Informatics, Centre for Preventive Medicine \& Digital Health Baden-Württemberg, University Medical Centre Mannheim, Mannheim, Germany

${ }^{4}$ Department of Neuroradiology, University Medical Centre Mannheim, Mannheim, Germany

${ }^{5}$ Institute for Clinical Radiology and Nuclear Medicine, University Medical Centre Mannheim, Mannheim, Germany

${ }^{6}$ DZHK (German Centre for Cardiovascular Research), Mannheim, Germany

Acknowledgements We thank all subjects who agreed to take part in the study, as well as our technical assistants Maria Moritz and Sabrina Kraemer (1st Department of Medicine, University Medical Centre Mannheim) for their help in acquiring lung function data.

Contributors All authors read and approved the final version of the manuscript. FT: writing the first draft of the manuscript, study design, data management, data analysis. MM: data analysis, statistical advice. KR: data collection, data management. AH: data management, data collection. JuS: data management, data analysis. JG: data analysis, study design, data management. JoS: study design, data analysis. IA: study design, revision of the first draft of the manuscript. MB: study design, revision of the manuscript. FJFH: study design, data analysis, revision of the manuscript. TG: data analysis, data management, revision of the first draft of the manuscript.

Funding Parts of our work received external funding from Ms Ester Knorr (Germany, private donation) and Markedsmodningsfonden (Denmark, public grant). Internal financial support was given by Heidelberg University (MEAMEDMA, internal grant).

Competing interests The following financial activities outside the submitted work exist: FT received travel support from Actelion, Berlin Chemie, Boehringer Ingelheim, Chiesi, Novartis, Mundipharma and TEVA, as well as speaker or consultation fees from AstraZeneca, Berlin Chemie, Boehringer Ingelheim, Bristol-Myers Squibb, Chiesi, GlaxoSmithKline, Roche, Novartis and Sanofi-Aventis. JoS received travel support and speaker fees from Boehringer Ingelheim, GlaxoSmithKline, Novartis and Roche. $\mathrm{MB}$ received speaker or consultation fees from Bayer, Boehringer Ingelheim, Daiichi Sankyo, Impulse Dynamics and Zoll Medical. IA received travel support as well as speaker or consultation fees from Abiomed, Bayer, Boehringer Ingelheim and St Jude Medical.

\section{Patient consent for publication Not required.}

Ethics approval The study protocol was approved by the Medical Ethics Committee II of the Medical Faculty Mannheim (Heidelberg University), compliant with the Declaration of Helsinki.

Provenance and peer review Not commissioned; externally peer reviewed.

Data availability statement All data relevant to the study are included in the article or uploaded as supplementary information. The models generated and analysed during the current study are available for download from an online repository (https://github.com/ftrinkmann).

Supplemental material This content has been supplied by the author(s). It has not been vetted by BMJ Publishing Group Limited (BMJ) and may not have been peer-reviewed. Any opinions or recommendations discussed are solely those of the author(s) and are not endorsed by BMJ. BMJ disclaims all liability and responsibility arising from any reliance placed on the content. Where the content includes any translated material, BMJ does not warrant the accuracy and reliability of the translations (including but not limited to local regulations, clinical guidelines, terminology, drug names and drug dosages), and is not responsible for any error and/or omissions arising from translation and adaptation or otherwise.

Open access This is an open access article distributed in accordance with the Creative Commons Attribution Non Commercial (CC BY-NC 4.0) license, which permits others to distribute, remix, adapt, build upon this work non-commercially, and license their derivative works on different terms, provided the original work is properly cited, appropriate credit is given, any changes made indicated, and the use is non-commercial. See: http://creativecommons.org/licenses/by-nc/4.0/.

\section{ORCID iDs}

Frederik Trinkmann http://orcid.org/0000-0001-5877-877X

Máté Maros http://orcid.org/0000-0002-1589-8699 


\section{REFERENCES}

1 Woodruff PG, Barr RG, Bleecker E, et al. Clinical significance of symptoms in smokers with preserved pulmonary function. N Engl J Med 2016;374:1811-21.

2 Trinkmann F, Henzler T, Saur J. Symptoms in smokers with preserved pulmonary function. N Engl J Med 2016:375:895-6.

3 Kjellberg S, Houltz BK, Zetterström 0, et al. Clinical characteristics of adult asthma associated with small airway dysfunction. Respir Med 2016;117:92-102.

4 Trinkmann F, Saur D, Roth K. Multiple breath washout (MBW) using sulfur hexafluoride - proof of concept in COPD. Eur Respir J 2016;48:PA3440.

5 Crisafulli E, Pisi R, Aiello M, et al. Prevalence of small-airway dysfunction among COPD patients with different gold stages and its role in the impact of disease. Respiration 2017;93:32-41.

6 O'Donnell DE, Revill SM, Webb KA. Dynamic hyperinflation and exercise intolerance in chronic obstructive pulmonary disease. Am J Respir Crit Care Med 2001;164:770-7.

7 Tzani P, Crisafulli E, Nicolini G, et al. Effects of beclomethasone/formoterol fixed combination on lung hyperinflation and dyspnea in COPD patients. Int J Chron Obstruct Pulmon Dis 2011;6:503-9.

8 Postma DS, Brightling C, Baldi S, et al. Exploring the relevance and extent of small airways dysfunction in asthma (ATLANTIS): baseline data from a prospective cohort study. Lancet Respir Med 2019;7:402-16.

9 Robinson PD, Latzin P, Verbanck S, et al. Consensus statement for inert gas washout measurement using multiple- and single- breath tests. Eur Respir J 2013:41:507-22.

10 Sullivan L, Forno E, Pedersen K, et al. Nitrogen back-diffusion during multiple-breath washout with 100\% oxygen. Eur Respir J 2017;50. doi:10.1183/13993003.006792017. [Epub ahead of print: 9 Sep 2017].

11 Kane M, Rayment JH, Jensen R, et al. Correcting for tissue nitrogen excretion in multiple breath washout measurements. PLoS One 2017;12:e0185553.

12 Nielsen JG. Lung clearance index: should we really go back to nitrogen washout? Eur Respir J 2014;43:655-6.

13 Lenherr N, Ramsey KA, Jost K, et al. Leaks during multiple-breath washout: characterisation and influence on outcomes. ERJ Open Res 2018:4. doi:10.1183/23120541.00012-2017. [Epub ahead of print: 23 Feb 2018].

14 Clemensen P, Christensen P, Norsk P, et al. A modified photo- and magnetoacoustic multigas analyzer applied in gas exchange measurements. J App/ Physiol 1994;76:2832-9.

15 Horsley AR, O'Neill K, Downey DG, et al. Closed circuit rebreathing to achieve inert gas wash-in for multiple breath wash-out. ERJ Open Res 2016;2. doi:10.1183/23120541.00042-2015. [Epub ahead of print: 22 Jan 2016].

16 Bell AS, Lawrence PJ, Singh D, et al. Feasibility and challenges of using multiple breath washout in COPD. Int J Chron Obstruct Pulmon Dis 2018;13:2113-9.

17 Yammine S, Lenherr N, Nyilas S, et al. Using the same cut-off for sulfur hexafluoride and nitrogen multiple-breath washout may not be appropriate. J Appl Physiol 2015;119:1510-2

18 Jensen R, Stanojevic S, Gibney K, et al. Multiple breath nitrogen washout: a feasible alternative to mass spectrometry. PLoS One 2013;8:e56868.

19 Nielsen N, Nielsen JG, Horsley AR. Evaluation of the impact of alveolar nitrogen excretion on indices derived from multiple breath nitrogen washout. PLoS One 2013;8:e73335.

20 Guglani L, Kasi A, Starks M. Difference between SF6 and N2 multiple breath washout kinetics is due to N2 back diffusion and error in N2 offset. J App/ Physiol 2018. doi:10.1152/japplphysiol.00326.2018

21 Quanjer PH, Tammeling GJ, Cotes JE, et al. Lung volumes and forced ventilatory flows. Report Working Party standardization of lung function tests, European community for steel and coal. official statement of the European respiratory Society. Eur Respir J Supp/ 1993;16:5-40.

22 Vogel J, Smidt U, Oscillometry I. Analysis of lung mechanics in general practice and clinic, epidemiological and experimental research. Frankfurt: PMI-Verlagsgruppe, 1994.

23 Trinkmann F, Götzmann J, Saur D, et al. Multiple breath washout testing in adults with pulmonary disease and healthy controls - can fewer measurements eventually be more? BMC Pulm Med 2017:17:185.
24 R Core Team. R: a language and environment for statistical computing. Vienna, Austria: R Foundation for Statistical Computing, 2017. https://www.R-project.org/

25 Rigby RA, Stasinopoulos DM. Generalized additive models for location, scale and shape (with discussion). J Royal Statistical Soc C 2005;54:507-54.

26 Lum S, Stocks J, Stanojevic S, et al. Age and height dependence of lung clearance index and functional residual capacity. Eur Respir J 2013;41:1371-7.

27 Alrumuh A, Gilchrist FJ, Bianco B, et al. P188 normative values of lung clearance index in children and adults using SF6 as washout gas. Journal of Cystic Fibrosis 2019;18:S111.

28 Horsley AR, Alrumuh A, Bianco B, et al. Lung clearance index in healthy volunteers, measured using a novel portable system with a closed circuit wash-in. PLoS One 2020;15:e0229300.

29 Fuchs SI, Eder J, Ellemunter $\mathrm{H}$, et al. Lung clearance index: normal values, repeatability, and reproducibility in healthy children and adolescents. Pediatr Pulmonol 2009:44:1180-5.

30 Verbanck S, Thompson BR, Schuermans D, et al. Ventilation heterogeneity in the acina and conductive zones of the normal ageing lung. Thorax 2012;67:789-95.

31 Houltz B, Green K, Lindblad A. Tidal N2 washout ventilation inhomogeneity indices in a reference population aged 7-70 years. European Respiratory Journal 2012;40:P3797.

32 Verbanck S, Paiva M. Gas mixing in the airways and airspaces. Compr Physiol 2011;1:809-34.

33 Dutrieue B, Vanholsbeeck F, Verbanck S, et al. A human acinar structure for simulation of realistic alveolar plateau slopes. J App/ Physiol 2000;89:1859-67.

34 Kim J, Heise RL, Reynolds AM, et al. Aging effects on airflow dynamics and lung function in human bronchioles. PLoS One 2017;12:e0183654.

35 Parraga G, Mathew L, Etemad-Rezai R, et al. Hyperpolarized 3 He magnetic resonance imaging of ventilation defects in healthy elderly volunteers: initial findings at 3.0 Tesla. Acad Radiol 2008;15:776-85.

36 Pellegrino R, Viegi G, Brusasco V, et al. Interpretative strategies for lung function tests. Eur Respir J 2005;26:948-68.

37 Cochrane GM, Prieto F, Clark TJ. Intrasubject variability of maximal expiratory flow volume curve. Thorax 1977:32:171-6.

38 Trinkmann F, Gawlitza J, Künstler M, et al. Small airway disease in pulmonary Hypertension-Additional diagnostic value of multiple breath washout and impulse oscillometry. J Clin Med 2018;7. doi:10.3390/jcm7120532. [Epub ahead of print: 09 Dec 2018].

39 Verbanck S, Schuermans D, Paiva M, et al. Small airway function improvement after smoking cessation in smokers without airway obstruction. Am J Respir Crit Care Med 2006;174:853-7.

40 Avramidou V, Hatziagorou E, Asterios K, et al. Is lung clearance index ( $\mathrm{LCl}$ ) affected by the severity of lung disease in CF? J Pulm Respir Med 2017;07.

41 Trinkmann F, Lenz SA, Schäfer J, et al. Feasibility and clinical applications of multiple breath wash-out (MBW) testing using sulphur hexafluoride in adults with bronchial asthma. Sci Rep 2020;10:1527.

42 Verbanck S, Paiva M, Schuermans D, et al. Relationships between the lung clearance index and conductive and acinar ventilation heterogeneity. J App/ Physiol 2012;112:782-90.

43 Quanjer PH, Weiner DJ, Pretto JJ, et al. Measurement of FEF25-75\% and FEF75\% does not contribute to clinical decision making. Eur Respir J 2014;43:1051-8.

44 Yammine S, Singer F, Abbas $C$, et al. Multiple-breath washout measurements can be significantly shortened in children. Thorax 2013;68:586-7.

45 Robinson PD, Stocks J, Aurora P, et al. Abbreviated multi-breath washout for calculation of lung clearance index. Pediatr Pulmonol 2013;48:336-43.

46 Grønbæk J, Hallas HW, Arianto L, et al. New time-saving predictor algorithm for multiple breath washout in adolescents. Pediatr Res 2016;80:49-53.

47 Hannon D, Bradley JM, Bradbury I, et al. Shortened lung clearance index is a repeatable and sensitive test in children and adults with cystic fibrosis. BMJ Open Respir Res 2014;1:e000031.

48 Saur J, Fluechter S, Trinkmann F, et al. Noninvasive determination of cardiac output by the inert-gas-rebreathing method--comparison with cardiovascular magnetic resonance imaging. Cardiology 2009;114:247-54. 